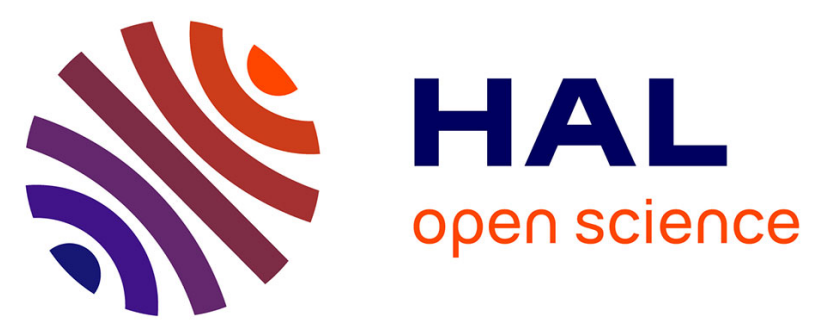

\title{
Perfectly matched layers as nonlinear coordinate transforms: a generalized formalization
}

\author{
Jean-Paul Hugonin, Philippe Lalanne
}

\section{To cite this version:}

Jean-Paul Hugonin, Philippe Lalanne. Perfectly matched layers as nonlinear coordinate transforms: a generalized formalization. Journal of the Optical Society of America. A Optics, Image Science, and Vision, 2005, 22 (9), pp.1844-1849. hal-00869730

\section{HAL Id: hal-00869730 \\ https://hal-iogs.archives-ouvertes.fr/hal-00869730}

Submitted on 4 Oct 2013

HAL is a multi-disciplinary open access archive for the deposit and dissemination of scientific research documents, whether they are published or not. The documents may come from teaching and research institutions in France or abroad, or from public or private research centers.
L'archive ouverte pluridisciplinaire HAL, est destinée au dépôt et à la diffusion de documents scientifiques de niveau recherche, publiés ou non, émanant des établissements d'enseignement et de recherche français ou étrangers, des laboratoires publics ou privés. 


\title{
Perfectly matched layers as nonlinear coordinate transforms: a generalized formalization
}

\author{
Jean Paul Hugonin and Philippe Lalanne \\ Laboratoire Charles Fabry de l'Institut d'Optique, Centre National de la Recherche Scientifique, \\ F-91403 Orsay cedex, France
}

Received January 21, 2005; accepted March 4, 2005

\begin{abstract}
A modified formulation of Maxwell's equations is presented that includes a complex and nonlinear coordinate transform along one or two Cartesian coordinates. The added degrees of freedom in the modified Maxwell's equations allow one to map an infinite space to a finite space and to specify graded perfectly matched absorbing boundaries that allow the outgoing wave condition to be satisfied. The approach is validated by numerical results obtained by using Fourier-modal methods and shows enhanced convergence rate and accuracy. (C) 2005 Optical Society of America

OCIS codes: $050.1960,050.1970,130.2790,050.1950,050.1940$.
\end{abstract}

\section{INTRODUCTION}

Advances in various branches of guided-wave photonics technologies have triggered considerable efforts to develop numerical tools for modeling a wide variety of dielectric waveguide structures that are not easily amenable to exact analytical studies. In these structures, as well as in other branches of computational electromagnetism, it is often required to satisfy outgoing wave conditions over semi-infinite half-spaces. Since its introduction in 1994, the so-called perfectly matched layer (PML) ${ }^{1}$ has been shown to be a remarkably effective means of satisfying outgoing wave conditions on finite computational grids. The concept of PMLs can be introduced in a variety of ways, using either split fields, ${ }^{1}$ anisotropic media, ${ }^{2}$ or complex coordinate stretching. ${ }^{3}$ Although introduced and used mainly for computations on finite-size grids with the finite-difference time-domain method, PMLs have also been considered with modal expansion techniques, including finite-difference schemes, ${ }^{4}$ exact modal expansions, ${ }^{5}$ and Fourier series, ${ }^{6,7}$ to cite only a few of them.

In a specific implementation, PML absorbers are often specified by an absorption profile, which is in general understood as a set of graded piecewise-constant PMLs stacked over a finite thickness. Examples are quadratic, ${ }^{1}$ quartic, $^{8}$ or somehow optimized ${ }^{9}$ absorption profiles. Through gradual variation, the objective is to minimize discretization errors and material-contrast reflections over a broad range of incidence. In this work, we propose a new formulation of PMLs relying on a complex nonlinear coordinate transform that maps the two semi-infinite half-spaces surrounding the investigated device geometry into a finite space. Theoretically, this mapping guarantees that the outgoing wave conditions are perfectly satisfied, since evanescent or propagative fields, incoming from the boundaries of the computational domain, are attenuated over an infinite distance before reaching the device. The mapping, which is implemented as a modification of the differential operators, appears as a generalization of more classical PML formulations, ${ }^{2,3}$ which are seen as a renor- malization of the permittivity and permeability distributions. In addition, the approach is particularly suitable for implementation with modal techniques, since it avoids the burdensome expansion of graded stacks of piecewiseconstant PMLs. In Section 2, we conceptually introduce nonlinear coordinate transforms as an efficient means to map semi-infinite spaces onto finite ones. Section 3 provides details on the numerical implementation of nonlinear transforms with Fourier-modal methods. In Section 4, the method is tested for two-dimensional geometries in terms of convergence performance and accuracy. While we chose to concentrate here on a two-dimensional example for the sake of simplicity, we did investigate some threedimensional geometries as well; they will be briefly mentioned, with examples provided through reference to recently published works.

\section{NONLINEAR TRANSFORM COORDINATE AND PERFECTLY MATCHED LAYERS}

Consider the situation depicted in Fig. 1(a), which shows a two-dimensional waveguide geometry used to illustrate the method. We assume that the waveguide is illuminated from the left-hand side by one of the guided modes of the waveguide. The permittivity and permeability depend on only the $x$ (transversal) and $z$ (longitudinal) coordinates. We assume that the structure as well as the incident field is invariant in the $y$ direction perpendicular to the figure. Both metallic and dielectric materials may be considered. Transverse-magnetic (TM) polarization is considered hereafter, the transverse-electric polarization case being straightforwardly deduced by using the symmetry of Maxwell's equations. Examples for the two polarization cases will be given in Section 4 .

We assume that the materials are magnetic with relative permeability $\mu(x, z)$ and anisotropic with a relative tensor of permittivity defined by $\varepsilon_{x x}(x, z)$ and $\varepsilon_{z z}(x, z)$, all the nondiagonal tensor coefficients being null for the sake 

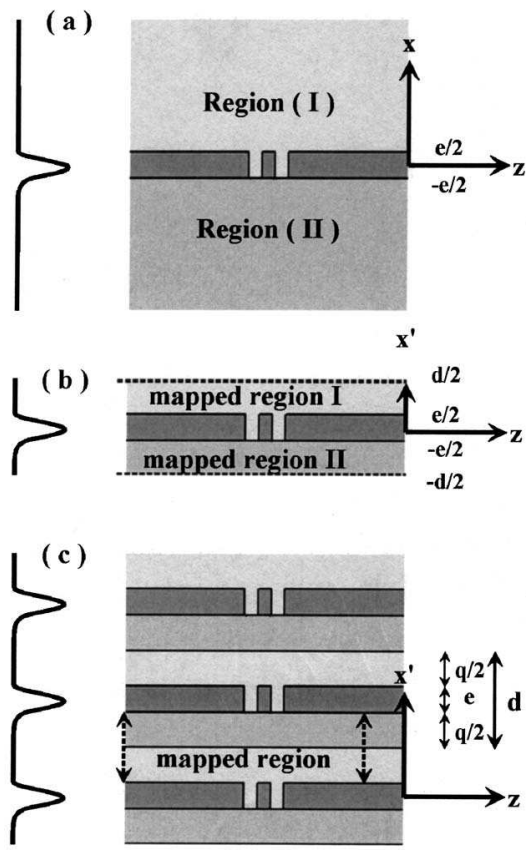

Fig. 1. Nonlinear transform for mapping an infinite space onto a finite one: (a) example of a diffraction problem "isolated in space" $(x \in]-\infty, \infty[)$, (b) equivalent diffraction problem in a bounded real space, $\left(x^{\prime} \in\right]-d / 2, d / 2[$ ), (c) same as (b) but with an artificial periodization of period $d$.

of simplicity. From the curl Maxwell's equations, after eliminating the electric-field components $E_{x}$ and $E_{z}$, we obtain

$$
\frac{\partial}{\partial z}\left(\frac{1}{\varepsilon_{x x}} \frac{\partial H_{y}}{\partial z}\right)+\frac{\partial}{\partial x}\left(\frac{1}{\varepsilon_{z z}} \frac{\partial H_{y}}{\partial x}\right)+k_{0}^{2} \mu H_{y}=0,
$$

where $H_{y}$ is the $y$ component of the magnetic field and $k_{0}$ is the modulus of the wavevector in a vacuum. We further assume that the permittivity and permeability in region (I) above the waveguide $(x>e / 2)$ and region (II) below the waveguide $(x<-e / 2)$ are independent of the $x$ coordinate. Although Fig. 1(a) depicts a situation for which these permittivities and permeabilities are independent of the $z$ coordinate, the analysis is not restricted to this specific case and encompasses geometries for which $\varepsilon_{x x}, \varepsilon_{z z}$, and $\mu$ may depend on $z$ in regions (I) and (II).

Our goal is to solve Eq. (1) while satisfying outgoing wave conditions in regions (I) and (II). For solving Eq. (1), many conventional numerical methods can be used; the specific method that we use will be described in Section 3 . For perfectly fulfilling the outgoing wave conditions, we use the fact that it is possible (1) to define an analytical continuation of $H_{y}$ in a suitable complex plane so that the incoming waves in regions (I) and (II) are prohibited and (2) to derive a simple complex coordinate transform that allows one to map this infinite complex plane into a finite segment of a new real space. To be more specific, we focus on region (I)first. In this region, the magnetic field, conveniently written as $H_{y}=H_{y}^{\text {out }}+H_{y}^{\text {in }}$, can be expanded ${ }^{10}$ in a modal basis of the form

$$
H_{y}^{\text {out }}=\sum_{p} a_{p} h_{p}(z) \exp \left(i k_{p} x\right),
$$

$$
H_{y}^{\text {in }}=\sum_{p} b_{p} h_{p}(z) \exp \left(-i k_{p} x\right)
$$

with $\operatorname{Re}\left(k_{p}\right)>0, \operatorname{Im}\left(k_{p}\right) \geqslant 0$, and $i^{2}=-1$. In Eqs. (2a) and (2b), the summations $\Sigma_{p}$ include a set of discrete modes and a continuum of evanescent ones. If the permittivities and permeability are independent of the $z$ coordinate, Eqs. (2) represent a plane-wave expansion. Clearly, $H_{y}^{\text {out }}$ and $H_{y}^{\text {in }}$ refer to outgoing and incoming modes, respectively. $H_{y}^{\text {out }}$ admits an analytical continuation ${ }^{11} H_{y}^{\text {out }}\left(X_{\mathrm{I}}, z\right)$ in the complex domain defined by $\operatorname{Re}\left(X_{\mathrm{I}}\right) \geqslant e / 2$ and $\operatorname{Im}\left(X_{\mathrm{I}}\right) \geqslant 0$, with $H_{y}^{\text {out }} \rightarrow 0$ as $X_{\mathrm{I}} \rightarrow \infty+i \infty$. This does not hold for $H_{y}^{\text {in }}\left(X_{\mathrm{I}}, z\right)$, since all propagative or evanescent modes in the summation of Eq. (2a) exponentially diverge as $\exp \left(-i k_{p} X_{\mathrm{I}}\right)$ for $X_{\mathrm{I}} \rightarrow \infty+i \infty$. Similar considerations hold for the field in region (II) for $\operatorname{Re}\left(X_{\mathrm{II}}\right) \leqslant-e / 2$ and $\operatorname{Im}\left(X_{\mathrm{II}}\right) \leqslant 0$.

Thus Eq. (1) remains valid in a complex space $X$ formed by the two semi-infinite domains, $X_{\mathrm{I}}$ and $X_{\mathrm{II}}$, and by a real segment $X_{\mathrm{III}}=x$ for $|x| \leqslant e / 2$. Instead of solving Eq. (1) in the domain $x \in]-\infty, \infty[$, we solve it rather in the complex plane $X$. More specifically, the integration of Eq. (1) is performed in a trajectory of $X$, which is conveniently mapped into a new finite real space $x^{\prime}$ of length $d\left(-d / 2<x^{\prime}\right.$ $<d / 2$ ) through a continuous complex coordinate transform $X=F\left(x^{\prime}\right)$ that must satisfy

$$
\begin{gathered}
F\left(x^{\prime}\right) \text { is real for }\left|x^{\prime}\right|<e / 2, \\
F\left(x^{\prime}\right) \rightarrow \infty+i \infty \quad \text { for } x^{\prime} \rightarrow d / 2, \\
F\left(x^{\prime}\right) \rightarrow-\infty-i \infty \quad \text { for } x^{\prime} \rightarrow-d / 2 .
\end{gathered}
$$

In the following, we simply chose $F\left(x^{\prime}\right)=x$ for $\left|x^{\prime}\right|<e / 2$. Thus the initial problem [Fig. 1(a)], which is defined in the domain $x \in]-\infty, \infty[$ and satisfies outgoing wave conditions in the clads, can be replaced by another, "equivalent" problem [Fig. 1(b)] with $H_{y}\left(x^{\prime}, z\right) \rightarrow 0$ for $\left|x^{\prime}\right| \rightarrow d / 2$ and $H_{y}\left(x^{\prime}, z\right)=H_{y}(x, z)$ for $\left|x^{\prime}\right|<e / 2$.

For expressing Eq. (1) in the new coordinate space, one needs only to specify the derivative $\mathrm{d} x^{\prime} / \mathrm{d} X=\left(\mathrm{d} F / \mathrm{d} x^{\prime}\right)^{-1}$. It is convenient to express this function in the form of the product of a continuous complex function $f\left(x^{\prime}\right)$ by a piecewise-constant complex function $f_{\mathrm{PML}}\left(x^{\prime}\right)$ :

$$
\mathrm{d} x^{\prime} / \mathrm{d} X=f\left(x^{\prime}\right) f_{\mathrm{PML}}\left(x^{\prime}\right) .
$$

Thus, in the new coordinate system $\left(x^{\prime}, z\right)$, Eq. (1) becomes

$$
\begin{array}{r}
\frac{\partial}{\partial z}\left[\left(\frac{1}{f_{\mathrm{PML}} \varepsilon_{x x}}\right) \frac{\partial H_{y}}{\partial z}\right]+\left(f \frac{\partial}{\partial x^{\prime}}\right)\left[\left(\frac{f_{\mathrm{PML}}}{\varepsilon_{z z}}\right)\left(f \frac{\partial}{\partial x^{\prime}}\right) H_{y}\right] \\
+k_{0}^{2}\left(\frac{\mu}{f_{\mathrm{PML}}}\right) H_{y}=0 .
\end{array}
$$

The net effect of the piecewise-constant function $f_{\mathrm{PML}}$ is a renormalization for the permittivity and permeability coefficients, such as for anisotropic-media PMLs, and the continuous-mapping contribution $f$ results in a modification of the differential operator $\partial(\cdot) / \partial x^{\prime}$. Equation (5) is general, and we believe that it can be solved with most frequency-domain methods that use field expansions on a regular function basis, such as finite-difference expansions or expansions relying on a basis of trigonometric 
functions that go to zero at the boundaries of the real segment $x^{\prime}$. As long as the basis is regular, i.e., does not encompass exponentially growing functions, incoming waves are prohibited and the outgoing wave condition is perfectly satisfied. The Fourier-modal method that we use hereafter to validate the approach comes from grating theories and, as explained in Ref. 6, amounts to solving the artificially periodized problem of Fig. 1(c). Exact solutions with a null field at $\left|x^{\prime}\right|<d / 2$ are expected when a sufficient number of Fourier harmonics are retained in the computation.

\section{IMPLEMENTATION WITH FOURIER- MODAL EXPANSION TECHNIQUES}

In this section, specifics of the coordinate transform used in Section 4 and its implementation with Fourier expansion techniques are considered. The literature on the analysis of photonics structures with Fourier expansion techniques is largely documented; see for instance the earlier Refs. 12-14 on the analysis of gratings and the more recent Refs. 6,15 , and 16 and references therein on the analysis of nonperiodic structures. Since they are not important for our understanding, all the steps leading to the formalism of Fourier-modal methods are not repeated hereafter. We rather refer to the work of Ref. 6 , where the numerical implementation of PMLs composed of uniform thin films with magnetic and anisotropic properties has been studied. The formalism developed hereafter appears simply as an extension of this earlier work.

With Fourier expansion methods, all the electromagnetic fields in the mapped space $x^{\prime}$ are expanded in Fourier series:

$$
\begin{aligned}
H_{y} & =\sum_{m=-M}^{M} U_{m}(z) \exp \left(i m K x^{\prime}\right), \\
\left(f_{\mathrm{PML}} \varepsilon_{x x}\right)^{-1} \frac{\partial H_{y}}{\partial z} & =\sum_{m=-M}^{M} V_{m}(z) \exp \left(i m K x^{\prime}\right),
\end{aligned}
$$

where $K=2 \pi / d$ and $M$ is the truncation rank. Let us denote by $\mathbf{K}_{x}$ a diagonal matrix with the $(m, m)$ element being $m K / k_{0}$ and by $\mathbf{E}, \mathbf{A}, \mathbf{B}$, and $\mathbf{F}_{x}$ the Toeplitz matrices associated with the Fourier coefficients of $\varepsilon_{z z}\left(x^{\prime}\right) / f_{\mathrm{PML}}$, $1 /\left[f_{\mathrm{PML}} \varepsilon_{x x}\left(x^{\prime}\right)\right], \mu\left(x^{\prime}\right) / f_{\mathrm{PML}}$, and $f$, respectively. In matrix notation, Eqs. (6) can be rewritten as

$$
\frac{\mathrm{d}}{\mathrm{d} z}\left(\begin{array}{c}
{[\mathbf{U}]} \\
{[\mathbf{V}]}
\end{array}\right)=\left(\begin{array}{cc}
\mathbf{0} & \mathbf{A}^{-1} \\
\mathbf{F}_{x} \mathbf{K}_{x} \mathbf{E}^{-1} \mathbf{F}_{x} \mathbf{K}_{x} & 0
\end{array}\right)\left(\begin{array}{l}
{[\mathbf{U}]} \\
{[\mathbf{V}]}
\end{array}\right),
$$

where $[\mathbf{U}]$ and $[\mathbf{V}]$ are vectors formed by the finite sets of the Fourier coefficients $U_{m}$ and $V_{m} \quad(m=-M,-M$ $+1, \ldots 0, \ldots, M)$ retained for the computation. If the geometry is composed of $z$-invariant sections as in the example of Fig. 1, the modes and their propagation constants in every section can be conveniently computed as eigenvectors and eigenvalues as we do in our implementation, and Eq. (7) reduces to Eq. (3) in Ref. 6, except that the matrix $\mathbf{K}_{x}$ has been replaced by the matrix $\mathbf{F}_{x} \mathbf{K}_{x}$. Note that the Fourier coefficients $f_{n}$ involved in $\mathbf{F}_{x}$ decrease as rapidly as $O\left(1 / n^{2}\right)$, since $f$ is a continuous function.
Clearly, many coordinate transforms may be investigated. We have not performed an extensive search for an optimum transform in terms of accuracy or convergence performance. Rather, our choice was motivated by its easy implementation with Fourier expansion techniques and has been inspired by an earlier work ${ }^{17}$ on the use of Fourier series for calculating bounded modes of rectangular core waveguides under the scalar-wave approximation with real nonlinear transforms. The chosen coordinate transform $X=F\left(x^{\prime}\right)$ is given in Appendix A. For our purpose, we need only to specify the function $\left(\mathrm{d} F / \mathrm{d} x^{\prime}\right)^{-1}$ $=f_{\mathrm{PML}}\left(x^{\prime}\right) f\left(x^{\prime}\right)$. The functions $f\left(x^{\prime}\right)$ and $f_{\mathrm{PML}}\left(x^{\prime}\right)$ are given by

$$
\begin{aligned}
f\left(x^{\prime}\right)= & 1 \quad \text { for }\left|x^{\prime}\right|<e / 2 \\
f\left(x^{\prime}\right)= & {\left[1-\gamma \sin ^{2}\left(\pi \frac{\left|x^{\prime}\right|-e / 2}{\mathrm{q}}\right)\right] \cos ^{2}\left(\pi \frac{\left|x^{\prime}\right|-e / 2}{\mathrm{q}}\right) } \\
& \text { for } e / 2<\left|x^{\prime}\right|<d / 2 \\
f_{\mathrm{PML}}\left(x^{\prime}\right)= & 1 \quad \text { for }\left|x^{\prime}\right|<e / 2 \\
f_{\mathrm{PML}}\left(x^{\prime}\right)= & f_{\mathrm{PML}} \quad \text { for } e / 2<\left|x^{\prime}\right|<d / 2
\end{aligned}
$$

where $q=d-e$ is the width of the mapped region and $\gamma$ and $f_{\mathrm{PML}}$ are complex parameters. The special case where no nonlinear mapping is performed will be referred to as $f=1$ hereafter. If $f_{\mathrm{PML}}=1$, no piecewise-constant mapping is performed.

Figure 2 shows the functions $f_{\mathrm{PML}}\left(x^{\prime}\right), f\left(x^{\prime}\right)$, and $F\left(x^{\prime}\right)$ along one period $d$ of the new coordinate system. Points defined by $x^{\prime}= \pm d / 2$ correspond to $\pm \infty$ in real space. For $\left|x^{\prime}\right| \rightarrow \pm d / 2,1 / f[1(1-\gamma)]\left\{q /\left[\pi\left(\left|x^{\prime}\right|-d / 2\right)\right]\right\}^{2}$, which corresponds to an infinite PML coefficient. A simple expression for the Fourier coefficients $f_{n}$ of $f\left(x^{\prime}\right)$ holds:

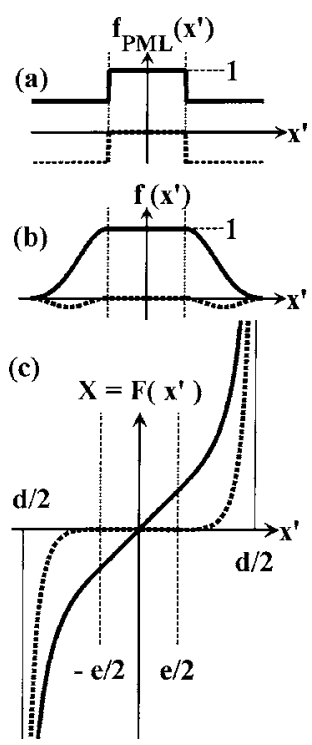

Fig. 2. Nonlinear coordinate transform: (a) function $f_{\mathrm{PML}}\left(x^{\prime}\right)$, (b) function $f\left(x^{\prime}\right)$, (c) function $X=F\left(x^{\prime}\right)$ for $\gamma=1 /(1-i)$ and $f_{\mathrm{PML}}=1$. Solid curves denote the real part, and dashed curves the imaginary part. 


$$
\begin{aligned}
f_{n}= & \delta_{n}-\frac{q}{2 d}(-1)^{n}\left[\left(1+\frac{\gamma}{4}\right) \operatorname{sinc}\left(\frac{n q}{d}\right)+\frac{1}{2} \operatorname{sinc}\left(\frac{n q}{d}-1\right)\right. \\
& \left.+\frac{1}{2} \operatorname{sinc}\left(\frac{n q}{d}+1\right)-\frac{\gamma}{8} \operatorname{sinc}\left(\frac{n q}{d}-2\right)-\frac{\gamma}{8} \operatorname{sinc}\left(\frac{n q}{d}+2\right)\right],
\end{aligned}
$$

where $\delta_{n}$ is equal to 1 if $n=1$ and to 0 otherwise and the function $\operatorname{sinc}(x)$ is $\sin (\pi x) /(\pi x)$.

\section{NUMERICAL EXAMPLES}

In this section, we illustrate the concepts developed in Section 2 on a simple double-slit two-dimensional geometry [see Fig. 3(a)]. This geometry has already been studied in Ref. 6 with PML for both TE and TM polarizations. It is composed of a corrugated waveguide with a 300-nm-wide core (refractive index 3.5) with claddings of refractive indices 2.9 for the substrate and 1 for the cover. Two identical 150-nm-large slits are etched into this waveguide. They are separated by a $150-\mathrm{nm}$-large spacer, and the slits are 300-nm deep. The geometry is illuminated by the $\mathrm{TM}_{0}$ (or $\mathrm{TE}_{0}$ ) fundamental guided mode from the left side, with a $975-\mathrm{nm}$ wavelength. A large fraction of the incident light being scattered into the claddings, this structure is relevant for studying the impact of the coordinate transform on the performance.

Figure 3 illustrates the effect of different piecewiseconstant PML and nonlinear coordinate transforms, as introduced in Section 2, which may be used for satisfying outgoing wave conditions. The numerical parameters used for the calculation are given in the figure caption. Figure 3(b) shows $\left|H_{y}\right|^{2}$ calculated with a complex coordinate transform $[\gamma=1 /(1-i)]$ with $f_{\mathrm{PML}}=1$. In the gray scale used for the plot, white zones correspond to null values of $\left|H_{y}\right|^{2}$. The field pattern exhibits the desirable behavior: While $\left|H_{y}\right|^{2}$ is intense in the waveguide core and in its vicinity, it vanishes as one reaches the boundaries $x^{\prime}$ $= \pm d / 2$ of the mapped space $(x= \pm \infty$ in the real space) represented by the dashed horizontal line. As discussed in Section 2, note that the observation of a perfectly null field distribution for $H_{y}$ and for its derivative at $x^{\prime}$ $= \pm d / 2$ guarantees that the field expansions in the claddings are composed only of outgoing waves. Figures $3(\mathrm{c})-3(\mathrm{f})$ show the transverse profiles of $\operatorname{Re}\left(H_{y}\right)$ computed in the claddings for several absorbers. In Fig. 3(c), the calculation is performed with a real coordinate transform $(\gamma=0)$ without PML $\left(f_{\mathrm{PML}}=1\right)$. As mentioned in Section 2, this configuration does not satisfy the outgoing wave condition, since the incoming propagative waves in the expansion of Eq. (2) are not attenuated. As shown in the figure, the field rapidly oscillates and is not null on the boundaries $x^{\prime}= \pm d / 2$. We have not been able to observe any convergence by increasing the truncation rank $M$ in this case. In Fig. 3(d), a complex nonlinear coordinate transform is used for $f_{\mathrm{PML}}=1$ and $\gamma=1 /(1-i)$ as in Fig. 3 (b). The far-field attenuation close to the boundaries $x^{\prime}$ $= \pm d / 2$ is very similar to that shown in Fig. 3(e) for a real coordinate transform $(\gamma=0)$ with PML $\left[f_{\mathrm{PML}}=1 /(1+i)\right]$. Note that the latter case corresponds to a usual uniform PML composed of an absorbing magnetic material extend-

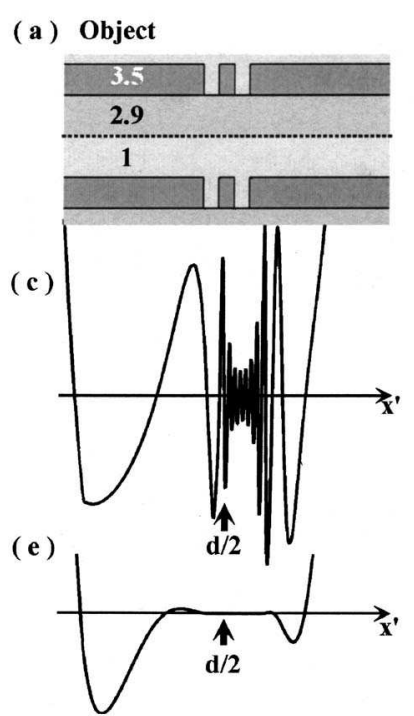

(b) $\mathrm{H}_{\mathrm{y}}$ field

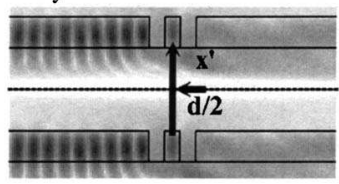

(d)

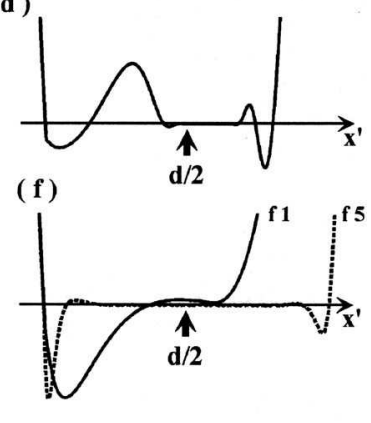

Fig. 3. Nonlinear and piecewise-constant coordinate transforms on a simple geometry problem. All plots are shown in the mapped space $\left(x^{\prime}, z\right)$, where $x^{\prime}=x$ if $f=1$. Numerical parameters are $d$ $=1.1 \mu \mathrm{m}, e=0.3 \mu \mathrm{m}$, and $M=100$. (a) Refractive index distribution of the double-slit geometry. (b) $\left|H_{y}\right|^{2}$ calculated for $\gamma=1 /(1$ $-i)$ and $f_{\mathrm{PML}}=1$. (c)-(f) Transverse profile of $\operatorname{Re}\left(H_{y}\right)$ [the horizontal axes correspond to the thick vertical line in (b), and the small vertical arrows correspond to $x^{\prime}=d / 2$ ]:

(c) real coordinate transform $(\gamma=0)$ without PML $\left(f_{\mathrm{PML}}=1\right)$,

(d) complex coordinate transform $[\gamma=1 /(1-i)]$ without PML $\left(f_{\mathrm{PML}}=1\right)$ as in $(\mathrm{b})$,

(e) real coordinate transform $(\gamma=0)$ with PML $\left[\left(f_{\mathrm{PML}}\right)^{-1}=1+i\right]$, (f) no nonlinear coordinate transform $(f=1)$ with $\left(f_{\mathrm{PML}}\right)^{-1}=1+i$ (solid curve) and $\left(f_{\mathrm{PML}}\right)^{-1}=5(1+i)$ (dashed curve).
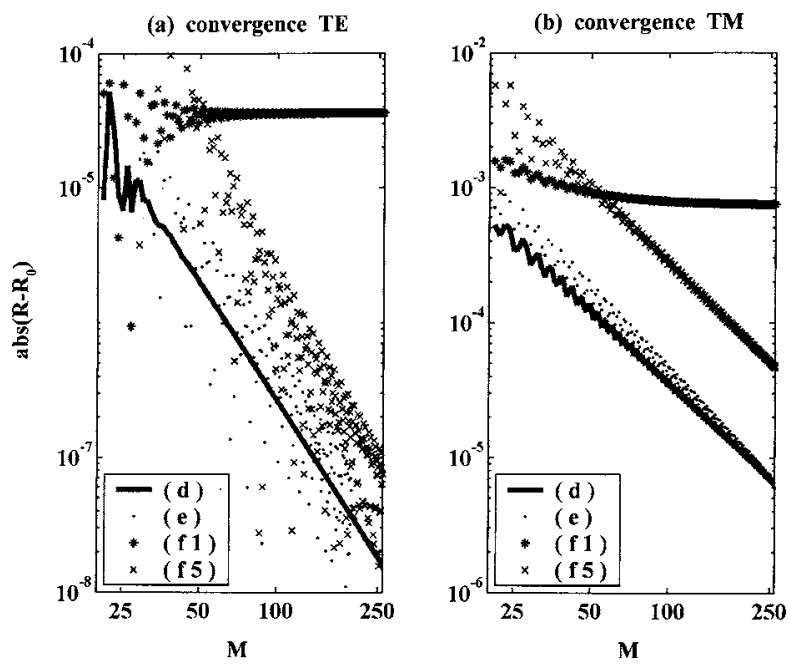

Fig. 4. Convergence and accuracy performance for the modal reflectivity $R$ associated into the different cases shown in Figs. 3(d) $-3(\mathrm{f})$. The extrapolated values for $R$ are $R_{0}=0.3952113445$ for TE polarization and 0.3554787 for TM polarization. The labels (d), (e), (f1), and (f5) refer to Figs. 3(d)-3(f) [with $\left.\left(f_{\mathrm{PML}}\right)^{-1}=1+i\right]$, and 3(f) [with $\left.\left(f_{\mathrm{PML}}\right)^{-1}=5(1+i)\right]$, respectively.

ing to infinity. In Fig. 3(f), no nonlinear coordinate transform $(f=1)$ is considered. The solid curve labeled $\mathrm{f} 1$ is obtained for $f_{\mathrm{PML}}=1 /(1+i)$. Clearly, the field is not null at the boundaries $x^{\prime}= \pm d / 2$. Again, this provides evidence that, in our periodized geometry, the field expansion in the claddings is not solely composed of outgoing waves. 
Quasi-null fields can be obtained by increasing the artificial period or by increasing the PML coefficient, as shown with the dashed curve obtained for $f_{\mathrm{PML}}=1 /[5(1+i)]$, labeled $\mathrm{f} 5$

In Fig. 4, the convergence rate and accuracy of previous piecewise-constant and nonlinear coordinate transforms are reported. With the complex coordinate transform, the computed modal reflected intensities, denoted $R$ hereafter, converge in a quasi-monotonic or smooth manner as the truncation rank $M$ increases. This suggests that extrapolation can be used to generate accurate values for $R$ and that these values can then be used to compute the errors obtained for small $M$ values. The anticipated errors do not correspond to the actual errors, as the latter could only be known if analytic or exact values are available. However, they still provide a useful measure of accuracy, since they vanish as more accurate results are generated. The general trends observed in Fig. 4 are rather expected or easily understood: The convergence rate is faster for TE $\left[O\left(M^{-3}\right)\right]$ than for TM $\left[O\left(M^{-2}\right)\right]$ and is lowered by use of large permittivity and permeability contrasts, such as those involved in the implementation of a PML with $f_{\mathrm{PML}}=1 /[5(1+i)]$ [case (f5)]. Concerning accuracy, the extrapolated values of the modal reflectivities for (d), (e), and (f5) are identical, with seven-(five-) digit accuracy for TE (resp. TM). However, they differ from that extrapolated from case (f1), for which the outgoing wave condition is poorly satisfied. The reason for this improvement is related to the fact that the PML approach does not map the entire infinite real space. Thus the computational results depend on the PML thickness and coefficient.

For the more computationally intensive threedimensional problems, the complex coordinate transform is straightforwardly implemented in Cartesian coordinates $(x, y, z)$. For an analytical integration along the $z$ direction, as in the previous two-dimensional case, the nonlinear transform is easily implemented as a product of two independent functions $F_{x}(x) F_{y}(y)$. In comparison with our earlier studies with uniform PMLs, ${ }^{6}$ we have observed an increase of the convergence rate or of the accuracy similar to those reported in Fig. 4. As shown by the numerical results obtained in Refs. 18-21, the approach has been successfully applied to different scattering problems including the calculation of the quality factor of micropillar cavities $^{18}$ and of photonic-crystal microcavities ${ }^{19}$ and the calculation of radiation losses in line-defect photonic-crystal waveguides. ${ }^{20}$ More recently, the use of the nonlinear real coordinate $(\gamma=0)$ described in this work has been successfully benchmarked for the calculation of the bounded modes (which are null at infinity) of a classical rib waveguide geometry. ${ }^{21}$

\section{CONCLUSION}

Two approaches allowing transforming an isolated diffraction problem [Fig. 1(a)] into an equivalent problem with null fields on the boundaries of a finite segment [Figs. 1(b) and 1(c)] have been presented. Outgoing wave conditions in the claddings can be satisfied with the help of a coordinate transform that replaces the field of the physical problem, which oscillates up to infinity, by its analytical continuation, which rapidly decreases toward zero in the mapped complex space.

The first approach consists in using a uniform PML with a real coordinate transform [method (e) in Figs. 3 and 4], and the second, which is more straightforward, consists in using a complex coordinate transform [method (d) in Figs. 3 and 4]. The numerical implementation of the real or complex coordinate transforms is simple, since it just amounts to multiplying the diagonal matrix associated with the space derivative by a Toeplitz matrix formed by the Fourier coefficients defined by Eq. (9). Moreover, since the transform coordinate is equal to the identity in the finite space corresponding to the waveguide region, the field of the unmapped region is left unchanged. These approaches have been shown to provide faster convergence rates and thus better accuracy than those already studied in Ref. 6 with a uniform PML [(f1) and (f5) in Figs. 3 and 4].

\section{APPENDIX A}

For the sake of completeness, we provide here the expression for the coordinate transform $X=F\left(x^{\prime}\right)$ that is associated with Eqs. (8) for $f_{\mathrm{PML}}=1$. Elementary algebra leads to

$$
\begin{aligned}
F\left(x^{\prime}\right)= & x^{\prime} \quad \text { for }\left|x^{\prime}\right|<e / 2, \\
F\left(x^{\prime}\right)= & \frac{x^{\prime}}{\left|x^{\prime}\right|}\left(\frac{e}{2}+\frac{q}{\pi(1-\gamma)}\left\{\tan \left(\pi \frac{\left|x^{\prime}\right|-e / 2}{q}\right)\right.\right. \\
& \left.\left.-\frac{\gamma}{\sqrt{1-\gamma}} a \tan \left[\sqrt{1-\gamma} \tan \left(\pi \frac{\left|x^{\prime}\right|-e / 2}{q}\right)\right]\right\}\right) \\
& \text { for } e / 2<\left|x^{\prime}\right|<d / 2 .
\end{aligned}
$$

\section{ACKNOWLEDGMENTS}

The authors acknowledge fruitful discussions in the past with Qing Cao on the use of nonlinear coordinate transforms in Fourier-modal methods and, more recently, with Ignacio del Villar, who has been partially involved in this work as a visiting graduate student in their Institute. Financial support from the European Network of Excellence NEMO is acknowledged.

Corresponding author: Jean-Paul.Hugonin@iota.upsud.fr.

\section{REFERENCES}

1. J. P. Bérenger, "A perfectly matched layer for the absorption of electromagnetic waves," J. Comput. Phys. 114, 185-200 (1994).

2. Z. Sacks, D. Kingsland, R. Lee, and J. Lee, "A perfectly matched anisotropic absorber for use as an absorbers boundary conditions," IEEE Trans. Antennas Propag. 43, 1460-1463 (1995).

3. W. C. Chew and W. H. Weedon, "A 3D perfectly matched medium from modified Maxwell's equations with stretched 
coordinates," Microwave Opt. Technol. Lett. 7, 599-604 (1994).

4. J. M. Elson and P. Tran, " $R$-matrix propagator with perfectly matched layers for the study of integral optical components," J. Opt. Soc. Am. A 16, 2983-2989 (1999).

5. P. Bienstman and R. Baets, "Advanced boundary conditions for eigenmode expansion models," Opt. Quantum Electron. 34, 523-540 (2002).

6. E. Silberstein, Ph. Lalanne, J. P. Hugonin, and Q. Cao, "On the use of grating theory in integrated optics," J. Opt. Soc. Am. A 18, 2865-2875 (2001).

7. Q. Cao, Ph. Lalanne, and J. P. Hugonin, "Stable and efficient Bloch-mode computational method for onedimensional grating waveguide," J. Opt. Soc. Am. A 19, 335-338 (2002)

8. J. C. Chen and K. Li, "Quartic perfectly matched layers for dielectric waveguides and gratings," Microwave Opt. Technol. Lett. 10, 319-323 (1995).

9. E. A. Marengo, C. M. Rappaport, and E. L. Miller, "Optimum PML ABC conductivity profile in FDTD," IEEE Trans. Magn. 35, 1506-1509 (1999).

10. E. Bahar, "Electromagnetic wave propagation in inhomogeneous multilayered structures of arbitrary varying thickness-generalized field transforms," J. Math. Phys. 14, 1024-1029 (1972).

11. D. Maystre, "General study of grating anomalies from electromagnetic surface modes," in Electromagnetic Surface modes, A. D. Boardman, ed. (Wiley, 1982), Chap. 17.

12. K. Knop, "Rigorous diffraction theory for transmission phase gratings with deep rectangular grooves," J. Opt. Soc. Am. 68, 1206-1210 (1978).

13. T. K. Gaylord and M. G. Moharam, "Analysis and application of optical diffraction by gratings," Proc. IEEE 73, 894-936 (1985)

14. F. Montiel and M. Nevière, "Differential theory of gratings: extension to deep gratings of arbitrary profile and permittivity through the $R$-matrix propagation algorithm," J. Opt. Soc. Am. A 11, 3241-3250 (1994).

15. Ph. Lalanne and E. Silberstein, "Fourier-modal method applied to waveguide computational problems," Opt. Lett. 25, 1092-1094 (2000).

16. J. Tervo, M. Kuittinen, P. Vahimaa, J. Turunen, T. Aalto, P. Heimala, and M. Leppihalme, "Efficient Bragg waveguidegrating analysis by quasi-rigorous approach based on Redheffer's star product," Opt. Commun. 198, 265-272 (2001).

17. S. J. Hewlett and F. Ladouceur, "Fourier decomposition method applied to mapped infinite domains: scalar analysis of dielectric waveguides down to modal cutoff," J. Lightwave Technol. 13, 375-383 (1995).

18. P. Lalanne, J. P. Hugonin, and J. M. Gérard, "Electromagnetic study of the $Q$ of pillar microcavities in the small limit diameter," Appl. Phys. Lett. 84, 4726-4728 (2004).

19. C. Sauvan, G. Lecamp, P. Lalanne, and J. P. Hugonin, "Modal-reflectivity enhancement by geometry tuning in photonic crystal microcavities," Opt. Express 13, 245-255 (2005).

20. C. Sauvan, P. Lalanne, J. C. Rodier, J. P. Hugonin, and A. Talneau, "Accurate modeling of line-defect photonic crystal waveguides," IEEE Photonics Technol. Lett. 15, 1243-1245 (2003).

21. J. P. Hugonin, P. Lalanne, I. Del Villar, and I. R. Matias, "Fourier modal methods for modeling optical dielectric waveguides," Opt. Quantum Electron. 37, 107-119 (2005). 